OPEN ACCESS

Edited by: Laura Vitiello,

San Raffaele Pisana (IRCCS), Italy

Reviewed by:

Marianna Capecci,

Marche Polytechnic University, Italy

Meg E. Morris,

La Trobe University, Australia

*Correspondence:

Siguang Li

siguangli@163.com

Lingjing Jin

lingjingjin@163.com

tThese authors have contributed equally to this work

Specialty section:

This article was submitted to

Neurorehabilitation,

a section of the journal

Frontiers in Neurology

Received: 30 April 2019 Accepted: 17 December 2019

Published: 28 January 2020

Citation:

Hu Y, Zhang $K$, Zhang T, Wang J, Chen F, Qin W, Tong W, Guan Q, He Y,

Gu C, Chen X, Kang UJ, Sun YE, Li S and Jin L (2020) Exercise Reverses

Dysregulation of T-Cell-Related

Function in Blood Leukocytes of

Patients With Parkinson's Disease.

Front. Neurol. 10:1389.

doi: 10.3389/fneur.2019.01389

\section{Exercise Reverses Dysregulation of T-Cell-Related Function in Blood Leukocytes of Patients With Parkinson's Disease}

\author{
Yong $\mathrm{Hu}^{1,2+}$, Kunshan Zhang ${ }^{3 \dagger}$, Tianyu Zhang ${ }^{1}$, Junbang Wang ${ }^{3}$, Fei Chen ${ }^{1}$, Wenting Qin ${ }^{4}$, \\ Weifang Tong ${ }^{1}$, Qiang Guan ${ }^{1}$, Yijing He ${ }^{1}$, Chunya $\mathrm{Gu}^{4}$, Xiaoyu Chen ${ }^{1}$, Un Jung Kang ${ }^{2}$, \\ Yi E. Sun ${ }^{3}$, Siguang $\mathrm{Li}^{3 *}$ and Lingjing Jin $^{1 *}$ \\ ${ }^{1}$ Department of Neurology, Shanghai Tongji Hospital, Tongji University School of Medicine, Shanghai, China, ${ }^{2}$ Department of \\ Neurology, Department of Neuroscience and Physiology, NYU Langone Health, The Marlene and Paolo Fresco Institute for \\ Parkinson's and Movement Disorders, Neuroscience Institute, New York, NY, United States, ${ }^{3}$ Stem Cell Translational \\ Research Center, Tongji Hospital, Tongji University School of Medicine, Shanghai, China, ${ }^{4}$ Department of Spine Surgery, \\ Shanghai Tongji Hospital, Tongji University School of Medicine, Shanghai, China
}

Parkinson's disease (PD) is a common neurodegenerative disease with movement and balance impairments. Although studies have reported improvement of motor symptoms with physical exercise, the mechanisms by which exercise is beneficial remains poorly understood. Our study addresses the exercise-induced changes to peripheral immune cells by interrogating the transcriptome of blood-derived leukocytes in PD patients before and after exercise. Patients attended $1 \mathrm{~h}$ exercise classes twice a week for 12 weeks. Leukocytes were collected at the beginning and end of the study for gene expression analysis by RNA-seq or quantitative real-time PCR. We correlated differentially expressed genes after exercise with clinical measures and analyzed the potential functions of gene changes with Kyoto Encyclopedia of Genes and Genomes pathway and Gene Ontology analysis. Exercise improved measures of movement and balance when compared with scores before the exercise program. Among the gene changes, Kyoto Encyclopedia of Genes and Genomes and Gene Ontology analysis suggests that T-cell receptor signaling, T-cell activation, and T-cell migration pathways were downregulated, while the T-cell receptor signaling pathway was the most significantly correlated with clinical measures. To further investigate T-cell-related changes in PD leukocytes, we reanalyzed the differentially expressed genes from publicly available microarray data and found that genes in the T-cell activation, differentiation, and migration pathways were upregulated in PD samples compared to controls in a time-dependent manner. Together, our findings suggest that exercise rehabilitation may improve movement and balance in PD patients by reversing the upregulated T-cell activation pathways associated with PD. This study was registered with the Chinese Clinical Trial Registry under ChiCTR-TRC-14004707. Registered on May 27, 2014.

Keywords: exercise, rehabilitation, Parkinson's disease, gene expression profile, inflammatory cytokine, $\mathrm{T}$ cell 


\section{INTRODUCTION}

Parkinson's disease (PD) is a common neurodegenerative disease characterized by loss of dopaminergic neurons in substantia nigra and accumulation of aggregated alpha-synuclein in the brain stem, spinal cord, and cortical regions (1). Movement and balance impairments, especially postural instability, adversely affect the daily function and quality of life of patients with PD (2). Although motor symptoms are alleviated with drug therapy, balance impairments are not optimally controlled by pharmacotherapy and require alternative approaches. Exercise has been shown to slow the deterioration of motor symptoms and prolong functional independence (2-4), improve movement $(3,4)$ and balance $(5)$, and decrease the incidence of falls (5-9). As such, exercise has been an integral part of PD management, although the underlying mechanisms by which exercise confers benefit remain controversial. Studies have suggested that exercise improves neurological function through neuroprotection (10, 11 ), improved neurogenesis, increased neuroregeneration (12, 13), and cardiorespiratory rehabilitation (14), although these findings have not been fully replicated (15). Accordingly, the mechanisms by which exercise is beneficial in patients with PD have not been fully elucidated.

Many neuron-intrinsic factors likely contribute to $\mathrm{PD}$ pathogenesis, including mitochondrial dysfunction, increased oxidative stress, and proteasomal dysfunction. In addition, increasing evidence suggests that perturbation of the immune system may play an important role in PD pathogenesis (1618). While central inflammation is widely recognized as a pathological hallmark of PD and may underlie neuronal death in the substantia nigra (19), mounting evidence suggests that peripheral inflammation contributes to the overall disease. In PD patients, blood-brain barrier impairment has been reported (20), and leukocytes have been identified near dopaminergic neurons in postmortem brain tissue from PD cases (21). In addition, many of the pathways implicated in neuronal dysfunction are also perturbed in peripheral blood cells from patients with $\mathrm{PD}$, including altered ubiquitin-proteasome and mitochondrial pathways and the presence of alpha-synuclein peptides $(22,23)$. In addition, evidence has suggested that peripheral inflammation plays a role in the early stages of disease initiation and progression, including the development of preclinical non-motor symptoms (19). These findings are supported studies manipulating peripheral immune cells in mouse models of PD, whereby inhibiting immune cell infiltration can attenuate dopaminergic neuron loss $(24,25)$. Together, these studies suggest that peripheral immune responses may contribute to the pathogenesis of $\mathrm{PD}$, and modulation of the immune system may be beneficial in the treatment of PD.

In healthy individuals, prolonged and regular physical exercise has been shown to promote an anti-inflammatory environment or attenuating the acute response to exercise $(26,27)$. Whether exercise has the same effect in PD patients has been largely unexplored, and the relationship between such effect and PD clinical symptoms remains unknown. To address these, we obtained gene expression profiles from peripheral leukocytes of $\mathrm{PD}$ patients before and after participating in an exercise program and correlated changes in gene expression profiles with clinical measures of movement and balance. In addition, we compared our experimental results with own analysis of a publicly available microarray dataset (GSE99039) and a microarray dataset from Parkinson's Progression Marker Initiative (PPMI).

\section{MATERIALS AND METHODS Clinical Study}

Patients with PD were recruited at the Shanghai Tongji Hospital by neurologists using the United Kingdom Parkinson's Disease Society Brain Bank clinical diagnostic criteria. Patients with confounding factors affecting the immune system were excluded. Two groups of patients on stable medication use were enrolled in this study, and they were asked to stay on the original medication dosage during the entire study. Blood samples of group 1 were used for RNA sequencing, while samples of group 2 were used to confirm the findings of group 1 with different methods (PCR and chemiluminescence immunoassay).

Participants were randomly allocated to tai chi (a kind of traditional Chinese martial arts) or multimodal exercise training (MET) group by researchers using a random numbers generator program, in a ratio of 1:1. The MET consisted of four exercise programs (core stability training, cycle ergometer, cross-obstacle training, and standing on ankle joint correcting board). Interventions were then performed as described before (28). The random number was delivered in a sealed envelope to each enrolled patient. Both groups attended exercise classes twice a week for 12 weeks. Assessments were completed by clinicians who were blinded to the mode of exercise intervention before and at the end of the 12 th weeks.

The effect of exercise on PD was assessed by Parkinson's Disease Rating Scale Motor Examination (UPDRSIII), stride length, gait speed, Timed Up and Go Test (TUG), and the Berg Balance Scale (BBS). Movement was assessed by UPDRSIII (29), stride length, gait speed, and TUG (30). Stride length and gait speed were assessed with a $10 \mathrm{~m}$ walk. BBS (31) was used to measure balance. Among these clinical measures, a higher score of UPDRSIII and TUG demonstrates worse symptoms that resulted in a lower score in stride length, gait speed, and BBS. The human experiment was approved by the Ethics Committee of Tongji Hospital, performed in accordance with the Declaration of Helsinki, and was registered with the Chinese Clinical Trial Registry under ChiCTR-TRC-14004707. Written informed consent was obtained from each participant.

\section{Blood Sample and RNA Isolation}

Peripheral venous blood samples in fasting state were collected by a standard procedure for complete blood counts and RNA isolation before the start and 48-72 h after the completion of the 12 weeks training program. Complete blood counts for white blood cell analysis were obtained from the clinical hematology laboratory by standard methods. Ethylenediaminetetraacetic acid was used as the anticoagulant for the isolation of peripheral blood leukocytes. The leukocytes were isolated and stored in TRIzol Reagent (Life Technologies) at $-80^{\circ} \mathrm{C}$ until RNA isolation was completed. Total RNA was extracted using TRIzol Reagent 
(Life Technologies) following the manufacturer's protocol for whole blood. RNA concentrations were assessed by running a small amount of each sample on Nanodrop 2000/2000C (Thermo Fisher Scientific). The RNA quality was checked using the Agilent 2100 Bioanalyzer.

\section{RNA Sequencing}

Total RNA was depleted of globin messenger RNA using the GLOBINclear-Human Kit (Life Technologies). Whole transcriptome libraries were made as outlined in the Ion Total RNA-Seq Kit v2 (Life Technologies). Selected libraries were diluted according to the final concentration of $10 \mathrm{pM}$ and clonally amplified using the Ion PI Template OT2 200 Kit v3 (Life Technologies). These amplified libraries were then purified and sequenced on the Ion Proton sequencer for a total of six sequencing runs (Ion PI Chip kit v2) (Life Technologies) according to the manufacturer's instructions.

\section{Transcriptome Analysis}

All reads were mapped to the human genome (hg19) by Tophat2 (v2.01). Unmapped reads were mapped again with bowtie2 (v2.10) in local mode. Alignment results were merged by the SAMtools. Normalized fragments per kilobase of transcript per million fragments mapped of genes in the RefGene annotation (2015 Feb) were estimated by Cuffquant (Cufflinks v2.21) with $[-b,-u]$ options followed by Cuffnormal (Cufflinks v2.21). Samples in two conditions (before and after exercise) were applied to paired student $t$-test to avoid individual differences. Significantly differentially expressed genes (DEGs, $P<0.05$ ) were used for enrichment analysis. Enrichment analysis including Gene Ontology (GO) analysis and Kyoto Encyclopedia of Genes and Genomes (KEGG) pathway analysis was performed by the Database for Annotation, Visualization, and Integrated Discovery. Paired centralized expression levels of each gene in each individual between two conditions were applied to principal component analysis. The microarray data of GSE99039 (22) was downloaded from the NCBI GEO database (http://www.ncbi. nlm.nih.gov/geo/) and analyzed by LIMMA. The microarray data of PD at baseline and 6 months after baseline was downloaded from the PPMI database (www.ppmi-info.org/data).

\section{Real-Time qPCR}

For confirmation of RNA-Seq findings, selected candidate genes were validated by quantitative real-time PCR (RT-qPCR). Total RNA was used to synthesize complementary DNA using Prinmascript RT Mastermix (Takara). All primers were designed using Primer3 (http://bioinfo.ut.ee/primer3/), synthesized by Sangon Biotech (see Table S1). BLAST searches were performed to confirm the specificity of the primer sequences. PCRs were carried out for 40 cycles $\left(95^{\circ} \mathrm{C}\right.$ for $15 \mathrm{~s}$ and $60^{\circ} \mathrm{C}$ for $60 \mathrm{~s}$ ) using QuantStudio 7 Flex Real-Time PCR System (Applied Biosystems). The PCR reaction mixture $(10 \mu \mathrm{l})$ contained 1 $\mu l$ of complementary DNA, specific primer sets, and Power SYBR Green PCR Master Mix (Applied Biosystems). The relative levels of selected genes were normalized to glyceraldehyde 3phosphate dehydrogenase.

\section{Cytokine Quantification in Plasma}

Cytokine levels of interleukin (IL)-6, IL-2R, and tumor necrosis factor (TNF) in sera of group 2 were quantified by chemiluminescence immunoassay with IMMULITE 1000 Immunoassay System (SIEMENS) according to the instructions of the manufacturer. All samples were measured by the average of two replicates of each sample. Concentrations below the detection limit were considered undetectable.

\section{Statistical Analysis}

Statistical analyses were carried out using SPSS (SPSS Inc., Chicago, Illinois, USA) version 20.0. Between-group differences in demographic and baseline variables were tested with nonparametric Mann-Whitney $U$-test and $\chi^{2}$-test for categorical variables and independent sample $t$-test for continuous variables. A normal distribution test was performed before each $t$-test that analyzed only normally distributed variables. Intervention effects on continuous outcome measures were compared by repeatedmeasures analysis of variance with two groups as the betweensubjects factor and time points as the within-subjects factor. Significance differences between the groups were tested with an independent sample $t$-test. Paired $t$-tests were used to examine within-group changes from baseline to the completion point of the 12 weeks exercise program. The significance level was set at $p$ $<0.05$, and all tests for significance were two-tailed.

\section{RESULTS}

\section{Exercise Improved Movement and Balance in Patients With PD}

From June 2014 to July 2015, 43 participants were consecutively randomized in group 1 (10 in the tai chi group and 11 in the MET group) and group 2 (11 in the tai chi group and 11 in the MET group) (Figure S1). Three of the 43 participants did not complete their assigned interventions (one in the tai chi group of group 2 and two in the MET group of group 2).

Table S2 summarizes the demographic characteristics, and no significant difference was observed between tai chi and MET group in all demographic characteristics. After a 12 weeks exercise program, patients from both groups demonstrated better performance in movement and balance compared with baseline regarding stride length, gait speed, TUG, and BBS (Figure 1A). They even achieved a lower score for UPDRS III compared with baseline, from $23.62 \pm 10.36$ to $18.10 \pm 10.05$ in the tai chi group and from $22.05 \pm 11.47$ to $15.10 \pm 6.78$ in the MET group (Figure 1A). In addition, no significant difference was observed between the two groups (tai chi and MET) in the five outcome measures (Figure 1A).

\section{Count of Leukocyte Types in PD Patients Remained Similar After Exercise}

Peripheral venous blood samples from patients with PD were collected before and after exercise to test the affection of exercise on the count of blood leukocytes types. The number of total white blood cells, neutrophils, and lymphocytes decreased mildly after exercise, while monocyte mildly increased. None of them experienced a significant change (Figure 1B). 
A

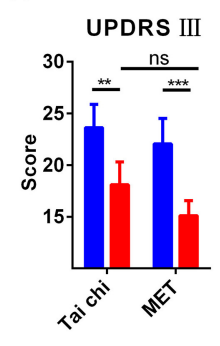

B

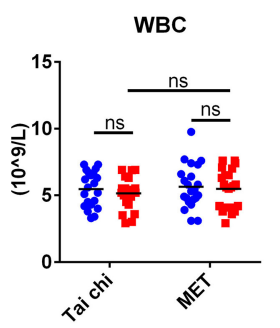

Before exercise

After exercise
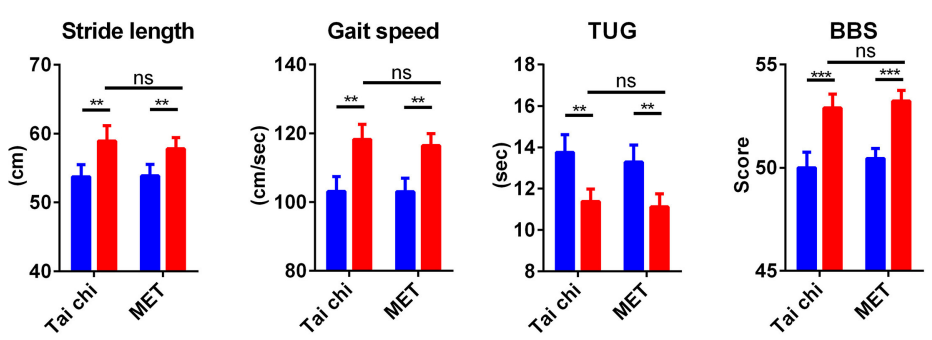

- Before exercise Neutrophils

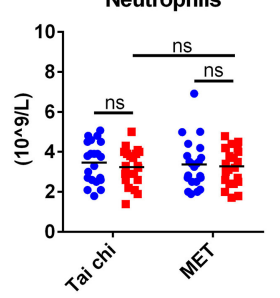

- After exercise
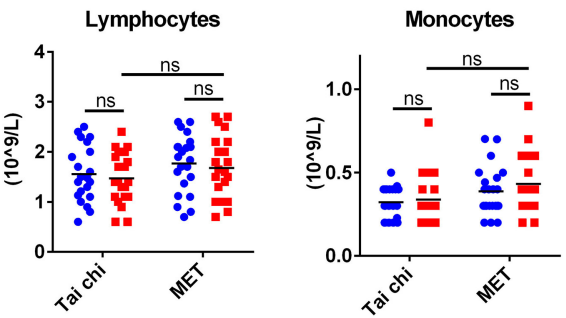

FIGURE 1 | Clinical measures and leukocyte response to exercise. (A) Exercise improved clinical measures for movement and balance in patients with Parkinson's disease (PD). Data are shown in mean \pm SEM. (B) Exercise did not change the count of leukocyte types in patients with PD. MET, multimodal exercise training. ${ }^{*} P<$ $0.05 ;{ }^{* *} P<0.01 ;{ }^{* \star *} P<0.001 ;$ ns, not significant.

\section{Exercise Regulated the Expression of Genes in Leukocytes of PD Patients}

To address the effect of exercise on leukocytes of PD patients, we performed RNA-Seq with leukocytes from 42 blood samples (21 PD patients of group 1 with every one taking a blood test before and after the exercise program), and the other 38 samples (19 PD patients of group 2) were used for RT-qPCR to confirm results of RNA-Seq. Principal component analysis of RNA-Seq data showed that samples in different conditions (before or after exercise) were separated (Figure 2A). However, consistent with clinical measures, the type of exercise (tai chi and MET) did not lead to a significant transcriptomic difference. Thus, in the following analysis, we combined the two groups (tai chi and MET) to explore the shared transcriptome differences driven by exercise. In the analysis of RNA-Seq data, 1,873 genes were identified as DEGs in the before- and after-exercise group (Figure 2B, Table S3). Among them, 1,453 DEGs had higher expression levels before exercise, while 417 DEGs had higher expression levels after exercise.

The most significantly downregulated gene after exercise was ODC1, which is functionally associated with PINK1, a causal gene responsible for hereditary recessive early-onset Parkinsonism (32). We also noted that CD3E, part of the TCRCD3 complex on the T-lymphocyte cell surface, was notably downregulated after exercise. The CD3E complex mediates signal transduction, resulting in T-cell activation (33). TRAF3, which was upregulated after exercise, is an essential constituent of several E3-ubiquitin-protein ligase complexes and might promote ubiquitination of target proteins (34). The significant downregulation of ODC1, CD3E, and upregulation of TRAF3 after exercise were confirmed by RT-qPCR in a separate validation cohort (group 2) (Table S4). In addition, we found that the expression of SNCA, GBA, PARK, LRRK2, PRKN, and PINK1, which are common genetic causes for PD, were not regulated by exercise.

Abnormal aggregation of $\alpha$-synuclein played a crucial role in the pathogenesis of synucleinopathies in PD $(23,35)$. In our previous study, AEP/PIKE-L axis was proved to regulate $\alpha$-synuclein-related etiopathological effects $(35,36)$. Our RNA-Seq data showed that, after exercise, both LGMN and AGAP2 exhibited a pattern indicating the amelioration of PD pathogenesis (Figure S2), in which AGAP2 was significantly upregulated after exercise.

\section{T-Cell-Related Programs Were Downregulated After Exercise}

To further understand the effect of physical exercise, we performed a functional analysis of DEGs. We observed 22 upregulated terms and 14 downregulated terms by KEGG pathway analysis (Figures 2C,D, Table S5). T-cell receptor signaling pathway and primary immunodeficiency pathway were highly enriched in downregulated genes, while the osteoclast differentiation pathway was the most enriched in upregulated genes after exercise. Consistent with the RNA-Seq results from group 1, members of the T-cell receptor signaling pathway (CD3E, GRAP2, FOS, and ZAP70) were significantly downregulated, which was confirmed by qPCR in group 2 (Table S4). These results indicated that the change in T-cell-related functions could be regulated by exercise.

Notably, the cytokine-cytokine receptor interaction pathway was enriched in both modulations (up- and downregulated) 
A

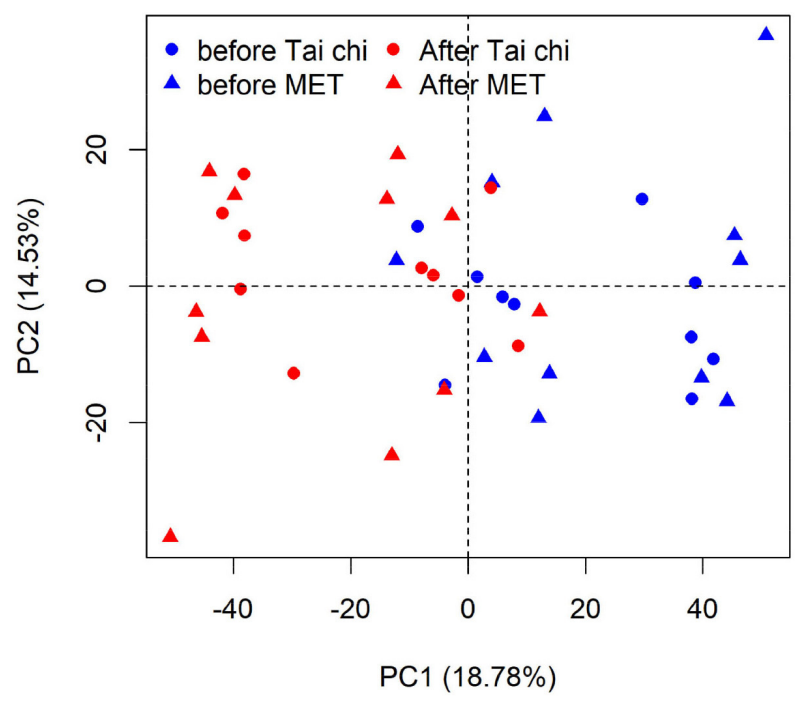

B Variables factor map (PCA)

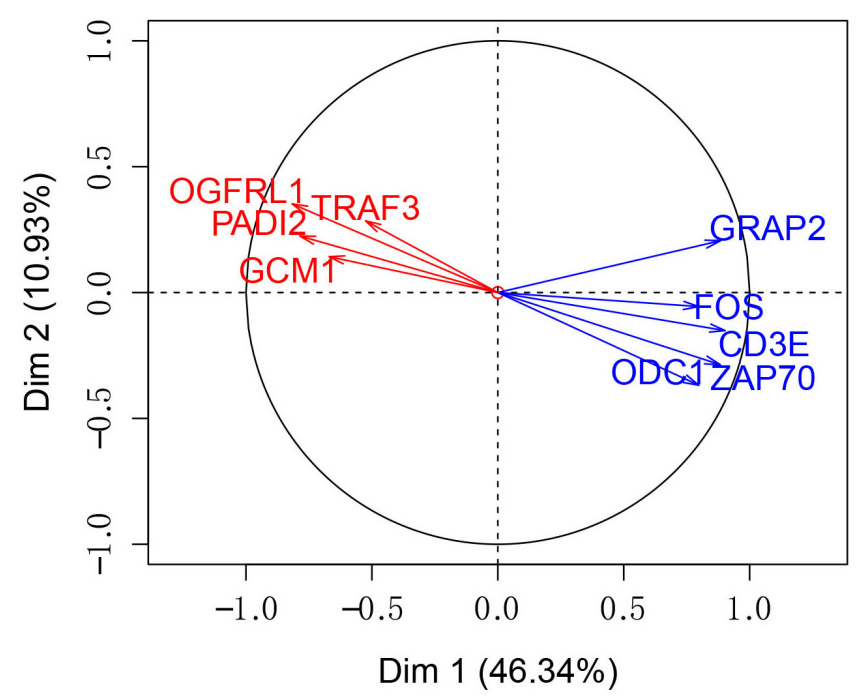

C

Up-regulated KEGG pathways after exercise

Fructose and mannose metabolism African trypanosomiasis

Fc gamma R-mediated phagocytosisHematopoietic cell lineageJak-STAT signaling pathway-

Toll-like receptor signaling pathwayNatural killer cell mediated cytotoxicityHepatitis C Cytokine-cytokine receptor interactionOsteoclast differentiation
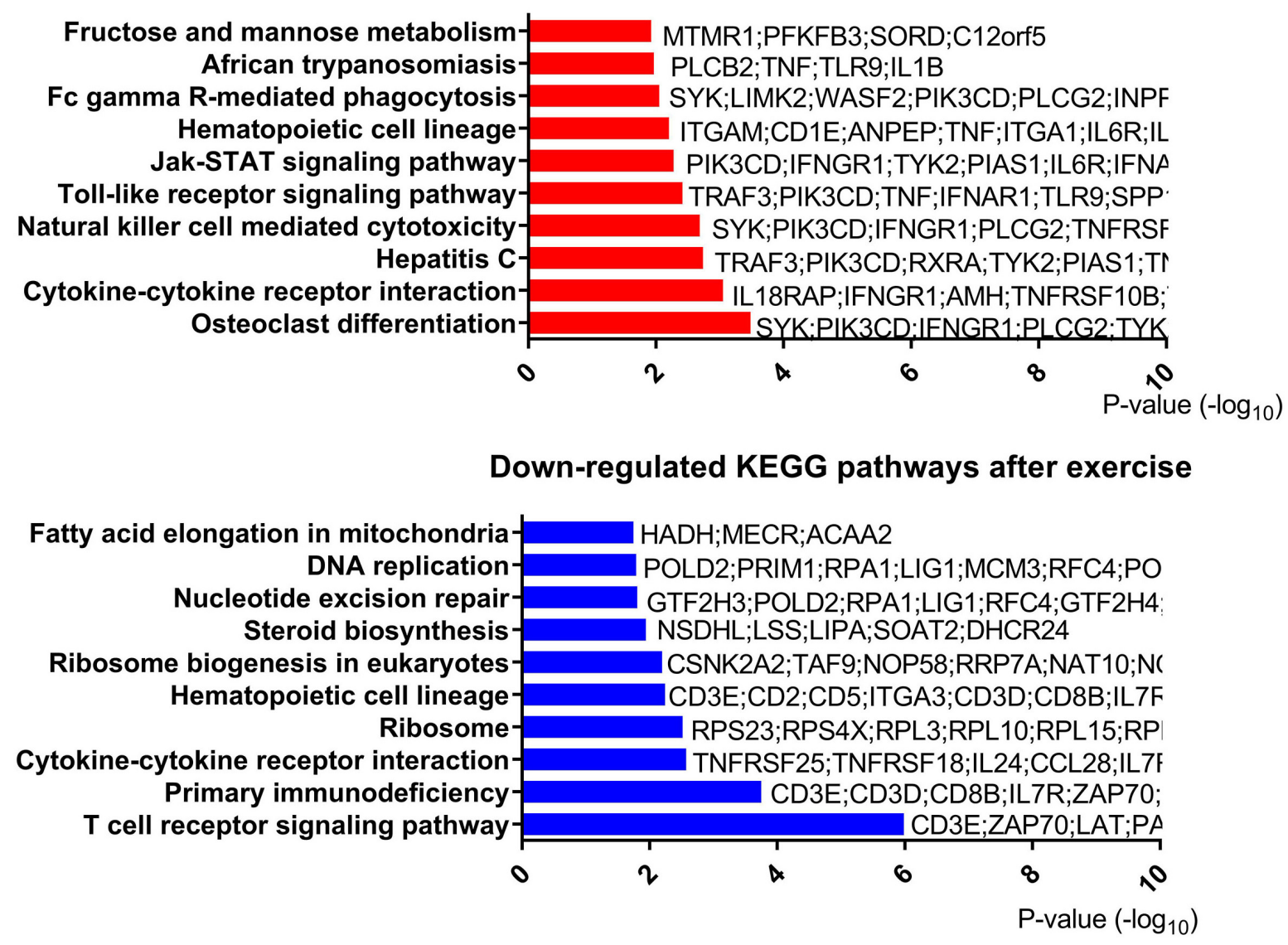

FIGURE 2 | Analyses of differentially expressed genes in the leucocytes of Parkinson's disease (PD) patients before and after exercise. (A) Principal component analysis of differentially expressed genes after centralized distinguishes between before and after exercise. PC, principal component; MET, multimodal exercise training. (B) Distinct gene groups characterized after exercise and before exercise groups on the basis of differential correlation with Dim 1 and Dim 2. Arrow tip denotes correlation coefficient of the distinct gene with each Dim. Dim, dimension. (C) Top 10 upregulated Kyoto Encyclopedia of Genes and Genomes (KEGG) pathways after exercise. (D) Top 10 downregulated KEGG pathways after exercise. 
with different genes (Figures 2C,D, Table S5). In these terms, we observed an upregulation of TNF and a downregulation of IL-2RA/B after exercise. To confirm the expression pattern of TNF and IL-2RA/B, we analyzed TNF and IL-2R levels in the serum of a different group of patients with PD (group 2, Figure S3). Similar to RNA-Seq results, an upregulation of proinflammatory TNF and a tendency for downregulation of IL$2 \mathrm{R}$ after exercise were observed in group 2. However, IL-6, a key inflammatory factor that was elevated in the serum of PD patients, showed no significant changes in the plasma protein and transcript levels. Furthermore, inflammation response transcription factor CEBPB (Figure S2) showed a downregulated trend after exercise, consistent with the downregulation of many inflammatory cytokines.

GO enrichment analysis highlighted the inflammation and T-cell-related pathways as well. We observed that "positive regulation of response to external stimulus" was enriched in the upregulated genes after exercise (Table S6).
In addition, for downregulated genes after exercise, the most significantly enriched terms were genes involved in positive regulation of leukocyte proliferation and transferase activity (Table S6). The downregulation of "positive regulation of leukocyte proliferation," which included T-cell activation regulating genes (CCL5, CD28, CD3E, IL23A, IL2RA, PRKCQ, TMIGD2, TNFSF9, ZAP70, and so on), was consistent with the dysfunction of T-cell receptor signaling pathway in KEGG pathway analysis. We observed that the term of SUMO ligase activity was enriched in the upregulated genes after exercise (Table S6), which included genes regulating the ubiquitin-proteasome system. We also found that genes related to mitochondrion organization, mitochondrial membrane, mitochondrial inner membrane, and mitochondrial fission were regulated after exercise (Table S6), but they were not among the most significantly regulated terms, indicating that the mitochondrion function was not tremendously affected by exercise.

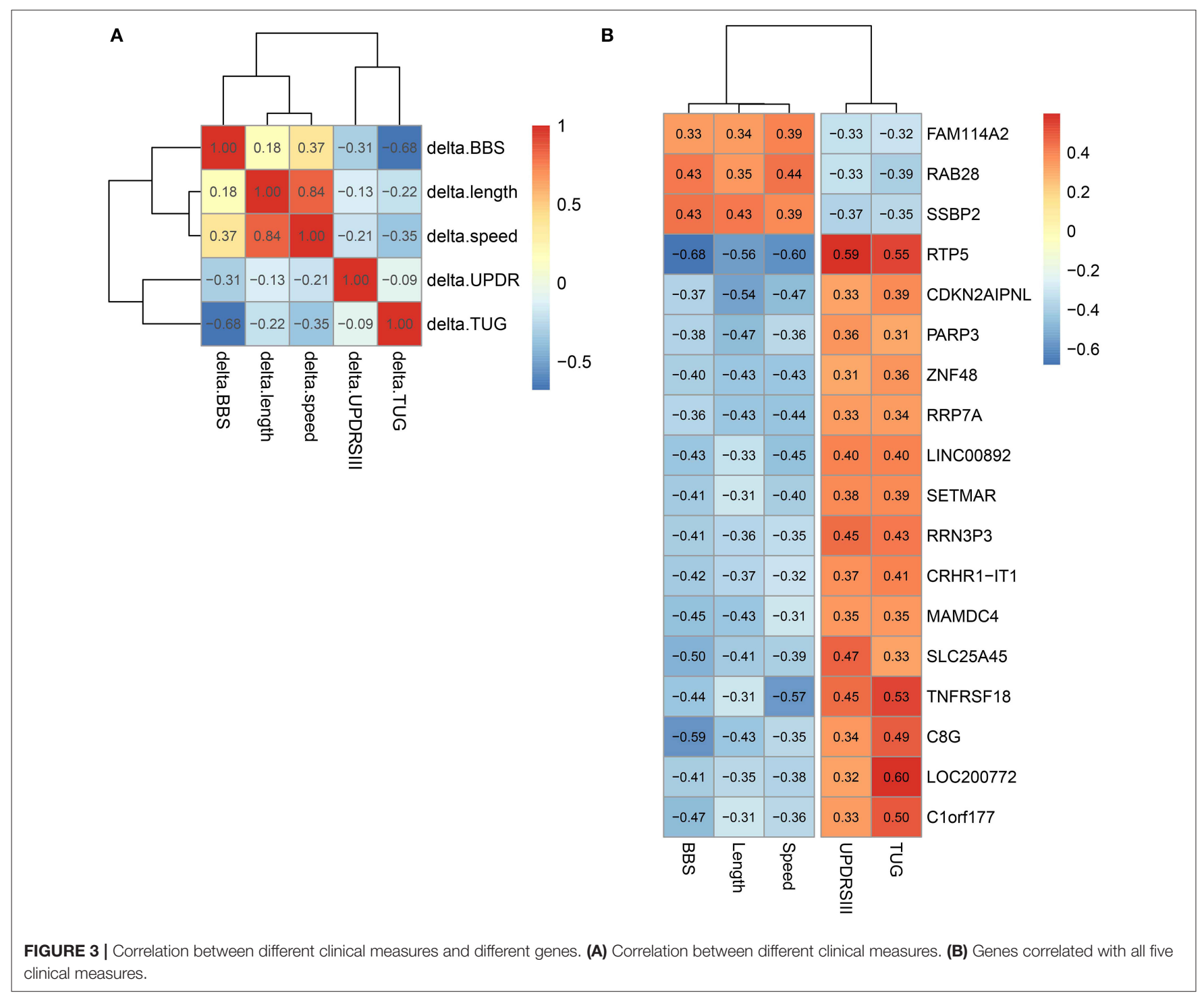




\section{Changes in the Genes for T-Cell Activation Correlated With Movement and Balance in PD}

We studied the relationship between clinical measures and transcriptome of blood leukocytes by Pearson correlation coefficients. The Pearson correlation test was conducted between the changes of five clinical measures after exercise, and a highly significant correlation (threshold $>0.5$ ) was identified between length and speed (both were measures for movement; Figure 3A). Moreover, TUG showed a highly significant correlation $(r=0.68)$ with BBS. Both measured the function of balance. We did not find a highly significant correlation between UPDRS and measures for movement and balance. Analysis of the BBS-correlated genes, which was measured for balance, showed that the most enriched signaling pathway was the T-cell receptor signaling pathway (Table 1). Interestingly, the T-cell receptor signaling pathway was also the enriched signaling pathways for length correlated genes (Table 1). For TUG speed, and UPDRSIII, very few correlated genes were identified (Table 1).

In all genes, 18 genes that significantly correlated with all the five clinical measures were identified (Figure 3B). Among them, we noted that the correlation between the clinical indicators and RTP5 and TNFRSF18 were the strongest, and both of them were downregulated after exercise. For RTP5, we found that it had a highly significant correlation (threshold $>0.5$ ) with all the five clinical parameters. But since it was a newly annotated gene, the function of RTP5 remained largely unknown. TNFRSF18, which encoded receptor, involved T-cell activation and interactions between activated T-lymphocytes and endothelial cells (37). The significant downregulation of TNFRSF18 (Table S4) was confirmed by qPCR in group 2 .

\section{Genes for T-Cell Activation and Differentiation Were Upregulated in PD}

Based on the change in T-cell-related pathways after exercise and its relationship with clinical measures, we hypothesized that T-cell-related functions, especially the T-cell receptor signaling pathway, were dysregulated in PD patients. To test this hypothesis, we reanalyzed publicly available microarray data (GSE99039) (22), which included peripheral blood from 205 PD patients and 233 control subjects. Genes (637 upregulated and 177 downregulated) were identified as DEGs in PD patients. Function enrichment analysis revealed that the T-cell receptor signaling pathway was among the most upregulated pathways in PD patients (Figure 4A, Table S7). We also noted that pathways for regulation of T-cell activation and differentiation were upregulated in PD patients (Figure 4A, Table S8). Among the DEGs, we found TNF receptor superfamily member 9 (TNFRSF9), which plays an essential role in T-cell activation, was upregulated. Both TNFRSF9 and TNFRSF18 belonged to TNF receptor superfamily. Interestingly, we found that genes for leukocyte transendothelial migration were upregulated in PD patients (Figure 4B).

To explore the change in T-cell-related function over time, we analyzed another microarray data from PPMI study downloaded on February 5th, 2019. Two hundred ninety-four PD patients
TABLE 1 | Correlation between Kyoto Encyclopedia of Genes and Genomes (KEGG) pathways and clinical measures.

\begin{tabular}{|c|c|c|c|}
\hline $\begin{array}{l}\text { KEGG } \\
\text { ID }\end{array}$ & pBH & $\begin{array}{l}\text { Odds } \\
\text { ratio }\end{array}$ & Term \\
\hline \multicolumn{4}{|c|}{ POSITIVE CORRELATION WITH BBS } \\
\hline 565 & 0.0170 & 10.31 & Ether lipid metabolism \\
\hline 4330 & 0.0179 & 7.72 & Notch signaling pathway \\
\hline 3008 & 0.0429 & 4.38 & Ribosome biogenesis in eukaryotes \\
\hline \multicolumn{4}{|c|}{ NEGATIVE CORRELATION WITH BBS } \\
\hline 4660 & 0.0000 & 8.77 & T cell receptor signaling pathway \\
\hline 5340 & 0.0000 & 15.28 & Primary immunodeficiency \\
\hline 4640 & 0.0002 & 5.87 & Hematopoietic cell lineage \\
\hline 5142 & 0.0112 & 3.64 & $\begin{array}{l}\text { Chagas disease (American } \\
\text { trypanosomiasis) }\end{array}$ \\
\hline 5200 & 0.0112 & 2.32 & Pathways in cancer \\
\hline 5322 & 0.0112 & 3.17 & Systemic lupus erythematosus \\
\hline 4514 & 0.0247 & 2.79 & Cell adhesion molecules (CAMs) \\
\hline 5020 & 0.0383 & 4.62 & Prion diseases \\
\hline 5330 & 0.0383 & 4.35 & Allograft rejection \\
\hline \multicolumn{4}{|c|}{ POSITIVE CORRELATION WITH LENGTH } \\
\hline 4380 & 0.0021 & 3.53 & Osteoclast differentiation \\
\hline 4662 & 0.0038 & 4.20 & B-cell receptor signaling pathway \\
\hline 4910 & 0.0045 & 3.00 & Insulin signaling pathway \\
\hline 5212 & 0.0045 & 4.05 & Pancreatic cancer \\
\hline 4210 & 0.0053 & 3.53 & Apoptosis \\
\hline 4370 & 0.0053 & 3.68 & VEGF signaling pathway \\
\hline 4664 & 0.0053 & 3.52 & Fc epsilon RI signaling pathway \\
\hline 4660 & 0.0053 & 3.05 & T-cell receptor signaling pathway \\
\hline 5223 & 0.0053 & 4.20 & Non-small cell lung cancer \\
\hline 4666 & 0.0053 & 3.22 & Fc gamma R-mediated phagocytosis \\
\hline 5120 & 0.0055 & 3.69 & $\begin{array}{l}\text { Epithelial cell signaling in Helicobacter } \\
\text { pylori infection }\end{array}$ \\
\hline 4722 & 0.0059 & 2.78 & Neurotrophin signaling pathway \\
\hline 4620 & 0.0080 & 2.94 & Toll-like receptor signaling pathway \\
\hline 5131 & 0.0081 & 3.64 & Shigellosis \\
\hline 5142 & 0.0081 & 2.87 & $\begin{array}{l}\text { Chagas disease (American } \\
\text { trypanosomiasis) }\end{array}$ \\
\hline 5214 & 0.0108 & 3.39 & Glioma \\
\hline 5145 & 0.0161 & 2.43 & Toxoplasmosis \\
\hline 4012 & 0.0166 & 2.79 & ErbB signaling pathway \\
\hline 4650 & 0.0166 & 2.35 & Natural killer cell mediated cytotoxicity \\
\hline 5220 & 0.0166 & 2.97 & Chronic myeloid leukemia \\
\hline 4520 & 0.0166 & 2.97 & Adherens junction \\
\hline 4330 & 0.0196 & 3.51 & Notch signaling pathway \\
\hline 4150 & 0.0297 & 3.13 & mTOR signaling pathway \\
\hline 4310 & 0.0297 & 2.10 & Wnt signaling pathway \\
\hline 5160 & 0.0307 & 2.16 & Hepatitis C \\
\hline 4720 & 0.0324 & 2.67 & Long-term potentiation \\
\hline 5211 & 0.0324 & 2.67 & Renal cell carcinoma \\
\hline 5140 & 0.0347 & 2.58 & Leishmaniasis \\
\hline 4810 & 0.0347 & 1.84 & Regulation of actin cytoskeleton \\
\hline 5221 & 0.0347 & 2.82 & Acute myeloid leukemia \\
\hline 450 & 0.0364 & 5.10 & Selenocompound metabolism \\
\hline
\end{tabular}

(Continued) 
TABLE 1 | Continued

\begin{tabular}{|c|c|c|c|}
\hline $\begin{array}{l}\text { KEGG } \\
\text { ID }\end{array}$ & pBH & $\begin{array}{l}\text { Odds } \\
\text { ratio }\end{array}$ & Term \\
\hline 4360 & 0.0438 & 2.02 & Axon guidance \\
\hline 4062 & 0.0478 & 1.78 & Chemokine signaling pathway \\
\hline 4930 & 0.0478 & 2.78 & Type II diabetes mellitus \\
\hline 520 & 0.0478 & 2.78 & $\begin{array}{l}\text { Amino sugar and nucleotide sugar } \\
\text { metabolism }\end{array}$ \\
\hline 4670 & 0.0478 & 2.02 & Leukocyte transendothelial migration \\
\hline 4140 & 0.0478 & 3.18 & Regulation of autophagy \\
\hline 10 & 0.0478 & 2.43 & Glycolysis/gluconeogenesis \\
\hline \multicolumn{4}{|c|}{ NEGATIVE CORRELATION WITH LENGTH } \\
\hline 4660 & 0.0000 & 4.08 & T-cell receptor signaling pathway \\
\hline 5340 & 0.0000 & 8.02 & Primary immunodeficiency \\
\hline 100 & 0.0010 & 9.15 & Steroid biosynthesis \\
\hline 4710 & 0.0134 & 5.80 & Circadian rhythm-mammal \\
\hline 4514 & 0.0156 & 2.35 & Cell adhesion molecules (CAMs) \\
\hline 4640 & 0.0229 & 2.55 & Hematopoietic cell lineage \\
\hline 4612 & 0.0229 & 2.66 & Antigen processing and presentation \\
\hline 3020 & 0.0229 & 4.11 & RNA polymerase \\
\hline 4650 & 0.0252 & 2.11 & Natural killer cell mediated cytotoxicity \\
\hline 514 & 0.0351 & 2.96 & Other types of O-glycan biosynthesis \\
\hline 5020 & 0.0358 & 3.28 & Prion diseases \\
\hline 5215 & 0.0363 & 2.23 & Prostate cancer \\
\hline 604 & 0.0392 & 4.90 & $\begin{array}{l}\text { Glycosphingolipid } \\
\text { biosynthesis-ganglio series }\end{array}$ \\
\hline 3008 & 0.0436 & 2.19 & Ribosome biogenesis in eukaryotes \\
\hline 5332 & 0.0480 & 2.73 & Graft-versus-host disease \\
\hline \multicolumn{4}{|c|}{ POSITIVE CORRELATION WITH SPEED } \\
\hline 230 & 0.0332 & 4.22 & Purine metabolism \\
\hline 1100 & 0.0480 & 1.95 & Metabolic pathways \\
\hline \multicolumn{4}{|c|}{ NEGATIVE CORRELATION WITH SPEED } \\
\hline 5322 & 0.0000 & 11.30 & Systemic lupus erythematosus \\
\hline 5020 & 0.0005 & 16.24 & Prion diseases \\
\hline 5150 & 0.0019 & 9.83 & Staphylococcus aureus infection \\
\hline 4610 & 0.0034 & 7.70 & $\begin{array}{l}\text { Complement and coagulation } \\
\text { cascades }\end{array}$ \\
\hline 4514 & 0.0260 & 3.84 & Cell adhesion molecules (CAMs) \\
\hline \multicolumn{4}{|c|}{ POSITIVE CORRELATION WITH UPDRS III } \\
\hline 534 & 0.0340 & 8.73 & $\begin{array}{l}\text { Glycosaminoglycan } \\
\text { biosynthesis - heparan sulfate }\end{array}$ \\
\hline 3008 & 0.0340 & 4.53 & Ribosome biogenesis in eukaryotes \\
\hline 340 & 0.0340 & 7.72 & Histidine metabolism \\
\hline 3410 & 0.0364 & 6.69 & Base excision repair \\
\hline 5200 & 0.0475 & 2.19 & Pathways in cancer \\
\hline 310 & 0.0475 & 4.88 & Lysine degradation \\
\hline 5222 & 0.0475 & 3.31 & Small cell lung cancer \\
\hline 330 & 0.0480 & 3.92 & Arginine and proline metabolism \\
\hline \multicolumn{4}{|c|}{ NEGATIVE CORRELATION WITH UPDRS III } \\
\hline 4621 & 0.0429 & 6.16 & NOD-like receptor signaling pathway \\
\hline 4380 & 0.0429 & 3.67 & Osteoclast differentiation \\
\hline 4664 & 0.0429 & 4.44 & Fc epsilon RI signaling pathway \\
\hline 4080 & 0.0429 & 2.61 & $\begin{array}{l}\text { Neuroactive ligand-receptor } \\
\text { interaction }\end{array}$ \\
\hline
\end{tabular}

POSITIVE CORRELATION WITH TUG

$\begin{array}{llll}3040 & 0.0346 & 4.77 & \text { Spliceosome }\end{array}$ with peripheral blood samples from baseline and 6 months after baseline were obtained from this microarray data. In the function enrichment, we found that $\mathrm{T}$-cell receptor signaling pathway was upregulated after 6 months (Figure 4C, Table S9). In addition, $\mathrm{T}$-cell activation and differentiation programs were also upregulated (Figure 4C, Table S10). In summary, these results showed that $\mathrm{T}$ cell was dysfunctional in $\mathrm{PD}$ patients and that leukocyte migration was activated in those patients.

\section{DISCUSSION}

Previous studies $(2,3)$ have shown that movement rehabilitation can improve movement and balance in patients with PD, which is consistent with our previous research. The present study also supports that patients with PD have different gene expression signatures before and after exercise. The gene expression changes suggest that exercise can modulate the abnormal immunity in patients with PD through T-cell-related function.

Although a consensus of the effectiveness of exercise in the management of PD has long been reached, the underlying pathomechanisms remain controversial. The immune system, especially the central immune system, has recently been recognized as a significant feature of PD pathophysiology. Chronic neuroinflammation, Lewy body inclusions, and loss of dopaminergic neurons in the substantia nigra parts compacta of the midbrain characterize PD pathology (38). Microglial activation and increased expression of inflammatory genes make up the in vivo evidence for neuroinflammation in PD patients. T-cell infiltration has been observed in affected brain areas in neurodegenerative diseases $(39,40)$, and the discovery of peripheral lymphocytes involving the progression of $\mathrm{PD}$ and hyperactivity in response to LPS stimulation $(17,41)$ shows that an inflammatory process is ongoing in the central nervous system and it may also be reflected in the peripheral circulation. For leukocyte microarray experiments, two studies have already been completed with more than 100 PD patients in each study $(22,42)$. Both studies focused on the molecular markers of PD, and they reported that functions related to metabolism, oxidation, and ubiquitination/proteasomal activity are dysregulated in PD patients. However, the studies did not pay much attention to the change in immune-related functions. Reanalysis of the first large microarray experiment highlighted modified neuroimmune signaling in blood cells from PD patients (43). Recently, DNA methylation alterations analysis of the second microarray experiment reveals that the most affected functions in PD by the methylation alterations are immune related, especially $\mathrm{T}$ cell activation and T-cell receptor signaling pathway (44). Based on the dysfunction of the immune system in PD patients, modulating neuroinflammation has been proven effective in reducing $\mathrm{T}$-cell infiltration and preventing the degeneration of dopaminergic neurons (24).

In this study, we tried to explore the mechanisms of exercise improving clinical symptoms of PD patients through RNASeq of circulating leukocyte collected before and after exercise. Previous studies showed exercise could induce a robust change in leukocyte function $(45,46)$. In this study, we found that patients 
A
$T$ cell related genes were upregulated in PD

KEGG

T cell receptor signaling pathway- VAV3;PTPN6;AKT1;RAF1;IKBKG;GRB2

regulation of T-helper 2 cell differentiation- $\quad$ PRKCZ;RARA;IL4R;

regulation of regulatory $T$ cell differentiation- HLA-G;LILRB2;IRF1;

alpha-beta T cell activation- LILRB1;HLA-E;C10orf54;SYK;PRKCZ;!

regulation of T cell cytokine production- C10orf54;PRKCZ;SASH3;TRAF6

T cell mediated cytotoxicity- LILRB1;HLA-C;HLA-E;HLA-G;HLA-B;r

regulation of CD8-positive, alpha-beta T cell activation- LILRB1; HLA-E; IRF1

positive regulation of T-helper 2 cell differentiation- $\quad$ PRKCZ;RARA;IL4R

regulatory $T$ cell differentiation-

positive regulation of $\mathrm{T}$ cell mediated cytotoxicity regulation of $\mathrm{T}$ cell differentiation-

$T$ cell cytokine production positive regulation of $\mathrm{T}$ cell differentiation regulation of $\mathrm{T}$ cell mediated cytotoxicity negative regulation of $\mathrm{T}$ cell proliferation

HLA-G;TGFB1;LILRB2;IRF1

HLA-C;HLA-E;HLA-G;HLA-B;HLA-F

ADAM8;HLA-G;CD74;SYK;LILRB2;E

C10orf54;SLC11A1;PRKCZ;SASH3;

ADAM8;CD74;HLA-G;IL4R;LILRB2;I

LILRB1;HLA-C;HLA-E;HLA-G;HLA-I

LILRB1;HLA-G;PTPN6;C10orf54;T

0

ら

P-value $\left(-\log _{10} \hat{)}\right.$

B

Leukocyte migration related genes were up-regulated in PD

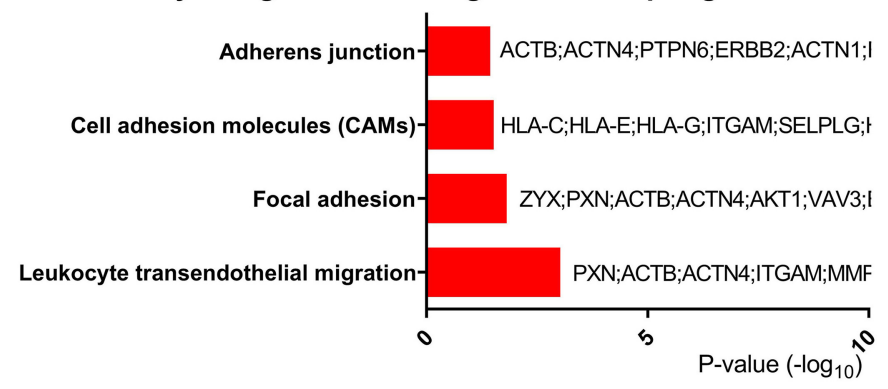

C

T cell related genes were upregulated in 6 months follow up

\section{KEGG}

T cell receptor signaling pathway- MAP2K2;NCK2;NFKBIE;

GO annotations

T cell differentiation involved in immune response- $B C L 3 ; N F K B I D ; R E L B ; Z C \Omega$ regulation of alpha-beta $T$ cell differentiation- NFKBID;ZAP70;ZC3H12A positive regulation of alpha-beta T cell differentiation- NFKBID;ZAP70;IL4R;AP3

T cell activation via T cell receptor contact with antigen bound to MHC- ICAM1;APBB1IP;HAVCR regulation of CD4-positive, alpha-beta T cell activation- NFKBID;ZC3H12A;LOXL: positive regulation of $T$ cell differentiation- BAD;NFKBID;ZAP70;IL4I regulation of alpha-beta $T$ cell activation- IRF1;NFKBID;ZAP70;TN CD4-positive, alpha-beta T cell differentiation- BCL3;NFKBID;RELB;ZC regulation of $T$ cell activation- $B A D ; C E B P B ; N C K 2 ; T N F$ positive regulation of $\mathrm{T}$ cell activation- $\mathrm{BAD}$;NCK2;TNFSF14;NI T-helper cell differentiation- $-B C L 3 ; N F K B I D ; R E L B ; Z C$ T cell differentiation- $\quad$ BAD;STK11;NCAPH2;BI

CD4-positive, alpha-beta T cell differentiation involved in immune-- $\quad B C L 3 ; N F K B I D ; R E L B ; Z C$ alpha-beta $T$ cell differentiation involved in immune response- $\quad B C L 3 ; N F K B I D ; R E L B ; Z$ alpha-beta $T$ cell activation involved in immune response- $B C L 3 ; N F K B I D ; R E L B ; Z$

1
0

P-value $\left(-\log _{10}\right)$

FIGURE 4 | Continued 
FIGURE 4 | T-cell-related genes were regulated in PD compared with control. (A) T-cell-related genes were upregulated in Parkinson's disease (PD). Only T-cell-related terms are presented here. The completed data of Kyoto Encyclopedia of Genes and Genomes (KEGG) pathway analysis and enriched Gene Ontology (GO) annotations are available in Tables S7, S8. (B) Leukocyte migration-related genes were upregulated in PD. The completed data of KEGG are available in Table S7. (C) T-cell-related genes were upregulated after 6 months in PD. The completed data of KEGG and enriched GO annotations are available in Tables S9, S10.

with PD had different gene expression signatures before and after a 12 weeks exercise program. We identified some PD-related biological function genes that were regulated after exercise, such as ODC1 and TRAF3. ODC1 is thought to be associated with mitochondrial dysfunction (47), while TRAF3 plays an important role in the ubiquitin-proteasome system (34). Furthermore, after exercise both LGMN and AGAP2, which have been proved to regulate $\alpha$-synuclein-related etiopathological effect, exhibited a pattern indicating amelioration of PD pathology. Besides the PD pathology-related pathways and biological function, the KEGG pathway and GO analysis highlighted that immune-related pathways and biological functions were regulated after exercise. The results support that exercise can modulate the abnormal gene expression patterns in leukocytes of PD patients.

DEG analysis also showed the TNFRSF18, a T-cell activation related gene was among the most downregulated genes after exercise and had the strongest correlation with clinical measures. Among those regulated pathways and biological processes, we found that the T-cell receptor signaling pathway was most involved before and after exercise, and it had a significant correlation with our clinical measures. In GSE99039 and PPMI microarray experiments, the T-cell receptor signaling pathway was upregulated in PD patients and further upregulated after 6 months, while T-cell receptor signaling pathway and Tcell-regulated genes were downregulated after exercise. It is suggested that exercise can ameliorate the aberrant of $\mathrm{T}$ cell-regulated genes in PD patients. In this study, we also found that genes for T-cell transendothelial migration were upregulated in PD patients. Previous studies have proved that brain-infiltrated $\mathrm{T}$ cell plays a crucial role in the degeneration of dopaminergic neurons $(24,39)$. These results support the hypothesis that exercise can affect the degeneration of dopaminergic neurons by modulating $\mathrm{T}$-cell activation and migration.

Another most affected pathway and biological process in our study was the cytokine-cytokine receptor interaction. In patients with $\mathrm{PD}$, it has been demonstrated that their serum TNF level, an area that appears to be more intensely studied than other inflammatory factors (48), was elevated. In this study, we observed that TNF was upregulated after exercise in both messenger RNA and serum protein levels, which was consistent with findings from studies of exercise in normal participants whose gene expression levels of TNF were increased after exercise $(49,50)$. Although exercise upregulates TNF, we did not find a significant correlation between the TNF level and any of the clinical measures. IL- 6 and IL-2R were elevated in the blood of $\mathrm{PD}$ patients and were significantly correlated with more severe symptoms (48). In this study, both IL-6 and IL-2R showed no significant change in serum after exercise, and they were not significantly correlated to clinical measures. These observations suggest that IL-6 and IL-2R may not be the key cytokines that control or mediate the effect of exercise in PD patients. More studies are required to identify key regulators that mediate the exercise effect.

In addition, epidemiological studies have suggested that persons with a history of moderate to vigorous exercise might have a reduction in PD risk (51). However, the research on the relationship between exercise and PD risk mainly focuses on neuroprotection. This study suggests that exercise plays a positive role in immune cells of PD patients, which may shed new light on the understanding of how exercise can reduce the risk of PD.

Although our RNA-Seq results were confirmed in the second group of $\mathrm{PD}$ patients, the results of this study might be vulnerable to bias. For example, the change in climate during the exercise could affect gene expression results. Moreover, we found that Tcell-related genes and functions were regulated after exercise, and they had a strong correlation with clinical measures. However, it remained unclear whether those changes directly improved the clinical indicators or whether they were just secondary responses to the improved clinical indicators. In addition, only 43 patients were enrolled in this study; larger-scale clinical researches with more measures would be more convincing to verify our findings.

\section{CONCLUSION}

In conclusion, our study found that exercise rehabilitation improved movement and balance in PD patients and changed the genes expression profile in their peripheral leukocytes. Based on pathway analysis, exercise decreased genes associated with Tcell activation, suggesting that exercise ameliorates PD symptoms by reducing peripheral inflammation. Our findings suggest that attenuating T-cell activation by exercise may be useful to complement pharmacotherapy for the control of PD symptoms.

\section{DATA AVAILABILITY STATEMENT}

The datasets generated for this study can be found in Gene Expression Omnibus (GEO), GSE124676.

\section{ETHICS STATEMENT}

This study was carried out in accordance with the recommendations of the Ethics Committee of Tongji Hospital with written informed consent from all subjects. All subjects gave written informed consent in accordance with the Declaration of Helsinki. The protocol was approved by the Ethics Committee of Tongji Hospital. 


\section{AUTHOR CONTRIBUTIONS}

LJ and SL contributed conception and design of the study. FC supervised the project. JW and YS prepared the experimental setup. TZ, JW, WQ, WT, QG, CG, XC, and YHe collected the data. $\mathrm{YHu}$ and $\mathrm{KZ}$ performed the statistical analysis. LJ, UK, SL, YS, YHu, and KZ discussed the findings. YHu and $\mathrm{KZ}$ wrote the first draft of the manuscript. All authors contributed to manuscript revision and read and approved the submitted version.

\section{FUNDING}

This study was supported by the National Key R\&D Program of China (2018YFC1314700 and 2018YFA0108000 to LJ), the National Natural Science Foundation of China (81572234 and 81873779 to LJ), the Shanghai Education Development Foundation, Shanghai Municipal Education Commission Shuguang Program (14SG21 to LJ), Priority of Shanghai Key Discipline of Medicine (2017ZZ02020 to LJ), and Fundamental Research Funds for the Central Universities (22120180111 to LJ).

\section{ACKNOWLEDGMENTS}

Some data used in the preparation of this article were obtained from the Parkinson's Progression Markers Initiative (PPMI) database (www.ppmi-info.org/data). For up-to-date information on the study, visit www.ppmi-info.org. PPMI-a public-private partnership-was funded by the Michael J. Fox Foundation for Parkinson's Research and funding partners, including Abbvie, Allergan, Avid, Biogen, BioLegend, Bristol-Myers Squibb, Celgene, Denali, GE Healthcare, Genentech, GlaxoSmithKline, Lilly, Lundbeck, Merck, Meso Scale Discovery, Pfizer, Piramal,

\section{REFERENCES}

1. Lees A, Hardy J, Revesz T. Parkinson's disease. Lancet. (2009) 373:2055-66. doi: 10.1016/S0140-6736(09)60492-X

2. Li F, Harmer P, Fitzgerald K, Eckstrom E, Stock R, Galver J, et al. Tai chi and postural stability in patients with Parkinson's disease. N Engl J Med. (2012) 366:511-9. doi: 10.1056/NEJMoa1107911

3. Tomlinson C, Patel S, Meek C, Herd C, Clarke C, Stowe R, et al. Physiotherapy versus placebo or no intervention in Parkinson's disease. Cochrane Database Syst Rev. (2013) 9:CD002817. doi: 10.1002/14651858.CD00 2817.pub4

4. Morris M, Perry A, Bilney B, Curran A, Dodd K, Wittwer J, et al. Outcomes of physical therapy, speech pathology, and occupational therapy for people with motor neuron disease: a systematic review. Neurorehabil Neural Repair. (2006) 20:424-34. doi: 10.1177/1545968305285092

5. Morris M, Iansek R, Kirkwood B. A randomized controlled trial of movement strategies compared with exercise for people with Parkinson's disease. Mov Disord. (2009) 24:64-71. doi: 10.1002/mds. 22295

6. Canning C, Sherrington C, Lord S, Close J, Heritier S, Heller G, et al. Exercise for falls prevention in Parkinson disease: a randomized controlled trial. Neurology. (2015) 84:304-12. doi: 10.1212/WNL.00000000000 01155
Prevail Therapeutics, Roche, Sanofi Genzyme, Servier, Takeda, Teva, UCB, Verily, and Voyager. We thank Dr. Bo Gu for critical reading of the manuscript.

\section{SUPPLEMENTARY MATERIAL}

The Supplementary Material for this article can be found online at: https://www.frontiersin.org/articles/10.3389/fneur. 2019.01389/full\#supplementary-material

Figure $\mathbf{S} 1$ | Flow diagram of the study.

Figure S2 | Gene expression change trend for AGAP2, LGMN, and CEBPB.

Figure S3 | The effect of exercise on inflammatory factors. (A) TNF- $\alpha$ level in blood plasma. (B) IL-2R level in blood plasma. (C) IL-6 level in blood plasma. Analyses were performed with paired $t$-tests. PD patients have higher levels of TNF- $\alpha$ after the training $(p<0.01)$. No significant difference is observed for IL2-R and IL-6. Red, increase after exercise; blue, decrease after exercise; black, no change after exercise.

Table S1 | Primer sequences used to quantify gene expression by RT-qPCR.

Table S2 | Clinical features of the study.

Table S3 | Differentially expressed genes list after exercise.

Table S4 | Validation of selected genes' expression in an independent cohort by RT-qPCR.

Table S5 | KEGG analysis for differentially expressed genes after exercise.

Table S6 | GO annotations for differentially expressed genes after exercise.

Table S7 | KEGG analysis for differentially expressed genes between Parkinson's disease and control.

Table S8 | GO annotations for differentially expressed genes between Parkinson's disease and control.

Table S9 | KEGG analysis for differentially expressed genes between new-onset Parkinson's disease and 6 months follow-up.

Table S10 | GO annotations for differentially expressed genes between new-onset Parkinson's disease and 6 months follow-up.

7. Fasano A, Canning C, Hausdorff J, Lord S, Rochester L. Falls in Parkinson's disease: a complex and evolving picture. Mov Disord. (2017) 32:1524-36. doi: $10.1002 / \mathrm{mds} .27195$

8. Morris M, Menz H, McGinley J, Watts J, Huxham F, Murphy A, et al. A randomized controlled trial to reduce falls in people with Parkinson's disease. Neurorehabil Neural Repair. (2015) 29:777-85. doi: $10.1177 / 1545968314565511$

9. Morris M, Taylor N, Watts J, Evans A, Horne M, Kempster P, et al. A home program of strength training, movement strategy training and education did not prevent falls in people with Parkinson's disease: a randomised trial. J Physiother. (2017) 63:94-100. doi: 10.1016/j.jphys.2017. 02.015

10. Tillerson J, Cohen A, Philhower J, Miller G, Zigmond M, Schallert T. Forced limb-use effects on the behavioral and neurochemical effects of 6-hydroxydopamine. J Neurosci. (2001) 21:4427-35. doi: 10.1523/JNEUROSCI.21-12-04427.2001

11. Hirsch M, van Wegen EEH, Newman M, Heyn PC. Exercise-induced increase in brain-derived neurotrophic factor in human Parkinson's disease: a systematic review and meta-analysis. Transl Neurodegener. (2018) 7:7. doi: 10.1186/s40035-018-0112-1

12. Ang E, Tai Y, Lo S, Seet R, Soong TW. Neurodegenerative diseases: exercising toward neurogenesis and neuroregeneration. Front Aging Neurosci. (2010) 2:25. doi: $10.3389 /$ fnagi.2010.00025 
13. Petzinger G, Fisher B, McEwen S, Beeler J, Walsh J, Jakowec MW. Exercise-enhanced neuroplasticity targeting motor and cognitive circuitry in Parkinson's disease. Lancet Neurol. (2013) 12:716-26. doi: 10.1016/S1474-4422(13)70123-6

14. Al-Jarrah M, Pothakos K, Novikova L, Smirnova I, Kurz M, Stehno-Bittel L, et al. Endurance exercise promotes cardiorespiratory rehabilitation without neurorestoration in the chronic mouse model of Parkinsonism with severe neurodegeneration. Neuroscience. (2007) 149:28-37. doi: 10.1016/j.neuroscience.2007.07.038

15. O’Dell S, Gross N, Fricks A, Casiano B, Nguyen T, Marshall JF. Running wheel exercise enhances recovery from nigrostriatal dopamine injury without inducing neuroprotection. Neuroscience. (2007) 144:1141-51. doi: 10.1016/j.neuroscience.2006.10.042

16. Schapira A, Jenner P. Etiology and pathogenesis of Parkinson's disease. Mov Disord. (2011) 26:1049-55. doi: 10.1002/mds.23732

17. Grozdanov V, Bliederhaeuser C, Ruf W, Roth V, Fundel-Clemens K, Zondler L, et al. Inflammatory dysregulation of blood monocytes in Parkinson's disease patients. Acta Neuropathol. (2014) 128:651-63. doi: 10.1007/s00401-014-1345-4

18. Maiti P, Manna J, Dunbar GL. Current understanding of the molecular mechanisms in Parkinson's disease: targets for potential treatments. Transl Neurodegener. (2017) 6:28. doi: 10.1186/s40035-0170099-Z

19. Su X, Federoff HJ. Immune responses in Parkinson's disease: interplay between central and peripheral immune systems. Biomed Res Int. (2014) 2014:275178. doi: 10.1155/2014/275178

20. Kortekaas R, Leenders K, van Oostrom J, Vaalburg W, Bart J, Willemsen A, et al. Blood-brain barrier dysfunction in parkinsonian midbrain in vivo. Ann Neurol. (2005) 57:176-9. doi: 10.1002/ana.20369

21. Hirsch EC. How to improve neuroprotection in Parkinson's disease? Parkinsonism Relat Disord. (2007) 13(Suppl. 3):S332-5. doi: 10.1016/S1353-8020(08)70026-9

22. Shamir R, Klein C, Amar D, Vollstedt E, Bonin M, Usenovic M, et al. Analysis of blood-based gene expression in idiopathic Parkinson disease. Neurology. (2017) 89:1676-83. doi: 10.1212/WNL.00000000000 04516

23. Sulzer D, Alcalay R, Garretti F, Cote L, Kanter E, Agin-Liebes J, et al. T cells from patients with Parkinson's disease recognize alpha-synuclein peptides. Nature. (2017) 546:656-61. doi: 10.1038/nature22815

24. Qin H, Buckley J, Li X, Liu Y, Fox T, 3rd, Meares G, et al. Inhibition of the JAK/STAT pathway protects against alpha-synuclein-induced neuroinflammation and dopaminergic neurodegeneration. $J$ Neurosci. (2016) 36:5144-59. doi: 10.1523/JNEUROSCI.4658-15.2016

25. Chung Y, Shin W, Baek J, Cho E, Baik H, Kim S, et al. CB2 receptor activation prevents glial-derived neurotoxic mediator production, BBB leakage and peripheral immune cell infiltration and rescues dopamine neurons in the MPTP model of Parkinson's disease. Exp Mol Med. (2016) 48:e205. doi: 10.1038/emm.2015.100

26. Gjevestad G, Holven K, Ulven SM. Effects of exercise on gene expression of inflammatory markers in human peripheral blood cells: a systematic review. Curr Cardiovasc Risk Rep. (2015) 9:34. doi: 10.1007/s12170-0150463-4

27. Rodriguez-Miguelez P, Fernandez-Gonzalo R, Almar M, Mejias Y, Rivas A, de Paz J, et al. Role of Toll-like receptor 2 and 4 signaling pathways on the inflammatory response to resistance training in elderly subjects. Age. (2014) 36:9734. doi: 10.1007/s11357-014-9734-0

28. Zhang T, Hu Y, Nie Z, Jin R, Chen F, Guan Q, et al. Effects of tai chi and multimodal exercise training on movement and balance function in mild to moderate idiopathic Parkinson disease. Am J Phys Med Rehabil. (2015) 94(10 Suppl. 1):921-9. doi: 10.1097/PHM.0000000000000351

29. Fahn S, Elton R, Members of the UPDRS Development Committee. Unified Parkinson's disease rating scale. In: Fahn S, Marsden C, Calne DB, and Goldstein M, editors. Recent Developments in Parkinson's Disease. Florham Park, NJ: Macmillan Health Care Information (1987). p. 153-63.

30. Podsiadlo D, Richardson S. The timed "Up \& Go": a test of basic functional mobility for frail elderly persons. J Am Geriatr Soc. (1991) 39:142-8. doi: 10.1111/j.1532-5415.1991.tb01616.x
31. Berg K, Wood-Dauphinee S, Williams J, Maki B. Measuring balance in the elderly: validation of an instrument. Can J Public Health. (1992) 83(Suppl. 2):S7-11.

32. Wasti A, Khan R, Jehan F, Anees Z, Karim H. Computational analysis of Parkinson's disease associated Pink1 gene: a neuroinformatics approach. Int J Adv Res. (2015) 3:645-52.

33. de Saint Basile G, Geissmann F, Flori E, Uring-Lambert B, Soudais C, Cavazzana-Calvo $M$, et al. Severe combined immunodeficiency caused by deficiency in either the delta or the epsilon subunit of CD3. J Clin Invest. (2004) 114:1512-7. doi: 10.1172/JCI2004 22588

34. Kayagaki N, Phung Q, Chan S, Chaudhari R, Quan C, O'Rourke K, et al. DUBA: a deubiquitinase that regulates type I interferon production. Science. (2007) 318:1628-32. doi: 10.1126/science.1145918

35. Kang S, Zhang Z, Liu X, Manfredsson F, He L, Iuvone P, et al. alpha-Synuclein binds and sequesters PIKE-L into Lewy bodies, triggering dopaminergic cell death via AMPK hyperactivation. Proc Natl Acad Sci USA. (2017) 114:1183-8. doi: 10.1073/pnas.1618627114

36. Zhang Z, Kang S, Liu X, Ahn E, Zhang Z, He L, et al. Asparagine endopeptidase cleaves alpha-synuclein and mediates pathologic activities in Parkinson's disease. Nat Struct Mol Biol. (2017) 24:632-42. doi: $10.1038 / \mathrm{nsmb} .3433$

37. Ronchetti S, Nocentini G, Riccardi C, Pandolfi PP. Role of GITR in activation response of $\mathrm{T}$ lymphocytes. Blood. (2002) 100:350-2. doi: 10.1182/blood-2001-12-0276

38. Kannarkat G, Tansey M. Role of the innate and adaptive immune system in the pathogenesis of PD. In: Thomas $\mathrm{M}$, editor. Inflammation in Parkinson's Disease. Cham: Springer International Publishing (2014). p. 75-103. doi: 10.1007/978-3-319-08046-8_3

39. Przedborski S. Neuroinflammation and Parkinson's disease. In: Koller WC, Melamed E, editors. Handbook of Clinical Neurology. New York, NY: Elsevier (2007). p. 535-51. doi: 10.1016/S0072-9752(07)83026-0

40. Brochard V, Combadiere B, Prigent A, Laouar Y, Perrin A, Beray-Berthat $\mathrm{V}$, et al. Infiltration of CD4+ lymphocytes into the brain contributes to neurodegeneration in a mouse model of Parkinson disease. J Clin Invest. (2009) 119:182-92. doi: 10.1172/JCI36470

41. Cao J, Li K, Shen YQ. Activated immune cells in Parkinson's disease. J Neuroimmune Pharmacol. (2011) 6:323-9. doi: 10.1007/s11481-0119280-9

42. Scherzer C, Eklund A, Morse L, Liao Z, Locascio J, Fefer D, et al. Molecular markers of early Parkinson's disease based on gene expression in blood. Proc Natl Acad Sci USA. (2007) 104:955-60. doi: 10.1073/pnas.0610204104

43. Soreq L, Israel Z, Bergman H, Soreq H. Advanced microarray analysis highlights modified neuro-immune signaling in nucleated blood cells from Parkinson's disease patients. J Neuroimmunol. (2008) 201:227-36. doi: 10.1016/j.jneuroim.2008.06.019

44. Wang C, Chen L, Yang Y, Zhang M, Wong G. Identification of potential blood biomarkers for Parkinson's disease by gene expression and DNA methylation data integration analysis. Clin Epigenet. (2019) 11:1-15. doi: 10.1186/s13148-019-0621-5

45. Connolly P, Caiozzo V, Zaldivar F, Nemet D, Larson J, Hung S, et al. Effects of exercise on gene expression in human peripheral blood mononuclear cells. J Appl Physiol. (2004) 97:1461-9. doi: 10.1152/japplphysiol.003 16.2004

46. Radom-Aizik S, Zaldivar F Jr, Leu S, Galassetti P, Cooper DM. Effects of $30 \mathrm{~min}$ of aerobic exercise on gene expression in human neutrophils. J Appl Physiol. (2008) 104:236-43. doi: 10.1152/japplphysiol.00872.2007

47. Simunovic F, Yi M, Wang Y, Macey L, Brown L, Krichevsky A, et al. Gene expression profiling of substantia nigra dopamine neurons: further insights into Parkinson's disease pathology. Brain. (2009) 132:1795-809. doi: 10.1093/brain/awn323

48. Nagamoto-Combs K, Combs CK. Proinflammatory chemical signaling: cytokines. In: Thomas $\mathrm{M}$, editor. Inflammation in Parkinson's Disease: Scientific and Clinical Aspects. Cham: Springer International Publishing (2014). p. 145-73. doi: 10.1007/978-3-319-08046-8_6

49. Tringali C, Scala L, Silvestri I, Vitale J, Scurati R, Michielon G, et al. Protective role of 17-beta-estradiol towards IL-6 leukocyte 
expression induced by intense training in young female athletes. $J$ Sports Sci. (2014) 32:452-61. doi: 10.1080/02640414.2013. 830190

50. Fernandez-Gonzalo R, De Paz J, Rodriguez-Miguelez P, Cuevas M, Gonzalez-Gallego J. Effects of eccentric exercise on toll-like receptor 4 signaling pathway in peripheral blood mononuclear cells. $J$ Appl Physiol. (2012) 112:2011-8. doi: 10.1152/japplphysiol.014 99.2011

51. Xu Q, Park Y, Huang X, Hollenbeck A, Blair A, Schatzkin A, et al. Physical activities and future risk of Parkinson disease. Neurology. (2010) 75:341-8. doi: 10.1212/WNL.0b013e3181 ea1597
Conflict of Interest: The authors declare that the research was conducted in the absence of any commercial or financial relationships that could be construed as a potential conflict of interest.

Copyright (c) $2020 \mathrm{Hu}$, Zhang, Zhang, Wang, Chen, Qin, Tong, Guan, He, Gu, Chen, Kang, Sun, Li and Jin. This is an open-access article distributed under the terms of the Creative Commons Attribution License (CC BY). The use, distribution or reproduction in other forums is permitted, provided the original author(s) and the copyright owner(s) are credited and that the original publication in this journal is cited, in accordance with accepted academic practice. No use, distribution or reproduction is permitted which does not comply with these terms. 